\title{
Preliminary clinical evaluation of meningococcal disease and bacterial meningitis by ultrasonic enhancement
}

\author{
R A Barnes, P Jenkins, W T Coakley
}

\begin{abstract}
Antigen detection in the urine and serum may be useful in the diagnosis of suspected meningococcal disease, especially after previous antibiotic treatment. Current test card procedures using commercial agglutination kits are often too insensitive to contribute to diagnosis. Diagnosis of meningococcal disease rose from $37 \%$ with the test card procedure to $\mathbf{7 4 \%}$ following ultrasonic enhancement. (Arch Dis Child 1998;78:58-60)
\end{abstract}

Keywords: meningococcal disease; diagnosis; ultrasound enhancement; antigen detection

Bacterial meningitis is a life threatening condition predominantly affecting young children and adolescents. Most cases in the United Kingdom are caused by Neisseria meningitidis but Streptococcus pneumoniae and group B streptococci may also be responsible. Haemophilus influenzae is now a rare cause since the introduction of the conjugate $\mathrm{Hib}$ vaccine in $1992 .{ }^{1}$ In addition to meningitis, $N$ meningitidis can cause septicaemia, often with profound endotoxic shock. Mortality is higher in the presence of septicaemia; meningococcal sepsis in the absence of meningitis carries the worst prognosis and often runs a rapid and fulminant course. Prompt diagnosis and interventive treatment is essential in these conditions. Traditionally, diagnostic techniques relied on demonstration of the causative organism in specimens of blood and cerebrospinal fluid (CSF). Blood cultures may take several days to yield a result. CSF specimens are no longer routinely obtained in many centres. Moreover, it is recommended that patients with suspected meningitis or meningococcal disease should receive antibiotics from their general practitioner before admission to hospital. ${ }^{2}$ This practice is life saving in some instances but severely limits the chances of obtaining a positive culture of the causative organism. ${ }^{3}$ This may have little impact on immediate patient management, but has implications in assessing the need for prophylaxis of close contacts and more importantly in determining antimicrobial sensitivities and epidemiological trends. Accurate knowledge of the distribution of the major serogroups (predominantly B and C in the United Kingdom) is an essential prerequisite for the development of an effective vaccine.

Molecular diagnosis using the polymerase chain reaction (PCR) is showing promise in the diagnosis of culture negative meningitis and meningococcal disease. ${ }^{4}$ However, this ap- proach is costly, time consuming, and usually specimens must be sent away to specialist laboratories. Antigen detection by latex agglutination is a useful adjunct since it can be performed rapidly and cheaply and may be positive even after antibiotic treatment. ${ }^{3}$ Commercially available kits exist for the diagnosis of infection caused by $N$ meningitidis groups $\mathrm{A}, \mathrm{B}$, C, Y, S pneumoniae, H influenzae, Escherichia coli, and group B streptococci. These tests were designed for use on CSF specimens and although now advocated for use on other body fluids such as blood and urine they are hampered by a lack of sensitivity. ${ }^{5}$

Immunoagglutination of coated microparticles can be enhanced by physical processes such as ultrasound. Microparticles in suspension in a non-cavitating ultrasonic standing wave concentrate within seconds at positions separated by half an acoustic wavelength. ${ }^{6}$ Previous studies at Cardiff have shown that the sensitivity of a number of diagnostic agglutination assays can be increased 250- to 2500 -fold using this technique. ${ }^{7}$

Improvement of detection of control antigens from commercially available diagnostic kits, and in some patient samples has been established and results presented elsewhere. ${ }^{8}$ Enhancements of 16- to 64-fold were seen with ultrasonic treatment compared to the standard test card procedure.

This study presents results from 114 individuals with a diagnosis of meningococcal disease, bacterial meningitis, or a range of related pathologies, as well as some controls. This preliminary study shows that ultrasonic enhancement significantly reduces the number of false negative results with latex agglutination while retaining specificity.

\section{Methods}

CLINICAL SAMPLES

Specimens (urine, serum, and/or CSF) were collected prospectively from 79 patients admitted to the University Hospital of Wales (UHW) with a clinical suspicion of bacterial meningitis or meningococcal septicaemia. These included specimens from 32 patients admitted during an outbreak of meningococcal disease at Cardiff University. An additional nine stored samples of CSF from eight patients collected between 1992 and 1993 were kindly provided by a colleague. The investigator performing the antigen testing was not informed of the likely diagnosis.

Patients were divided into four sets (table 1) as follows:

(1) Set 1 comprised 32 patients with microbiologically documented meningitis/sepsis 
Table 1 Patient characteristics and specimens

\begin{tabular}{|c|c|c|c|c|c|c|}
\hline & Disease category (patients) & Age range & $C S F$ & Serum & $\begin{array}{l}\text { Urine } \\
\text { (neat) }\end{array}$ & $\begin{array}{l}\text { Urine } \\
\text { (concentrated) }\end{array}$ \\
\hline \multirow[t]{4}{*}{ Set $1, n=32$} & Meningococcal disease, $n=24$ & 8 months to 40 years & 11 & 11 & 9 & 4 \\
\hline & Pneumococcal meningitis, $n=5$ & 8 months to 54 years & 1 & 0 & 5 & 4 \\
\hline & Pneumococcal sepsis, $n=2$ & 38 years and 40 years & 0 & 0 & 2 & 1 \\
\hline & Group B streptococcus meningitis, $n=1$ & 2 months & 0 & 0 & 1 & 0 \\
\hline Set $2, n=19$ & Clinical meningococcal sepsis, $\mathrm{n}=19$ & 5 months to 34 years & 2 & 9 & 13 & 8 \\
\hline \multirow[t]{3}{*}{ Set $3, n=35$} & Possible meningitis, $\mathrm{n}=30$ & 1 year to 3 years & 0 & 31 & 2 & 0 \\
\hline & Lobar pneumonia, $\mathrm{n}=3$ & 22 years to 41 years & 0 & 0 & 3 & 0 \\
\hline & OPSI, $\mathrm{n}=2$ & 30 years, 45 years & 0 & 1 & 2 & 1 \\
\hline \multirow{2}{*}{ Set $4, n=23$} & Controls, $n=10$ & & 0 & 8 & 10 & 10 \\
\hline & Unrelated conditions, $\mathrm{n}=13$ & & 2 & 8 & 5 & 4 \\
\hline Total = 109 & & & 16 & 68 & 52 & 32 \\
\hline
\end{tabular}

caused by organisms represented in the diagnostic kit. Twenty four patients had microbiologically documented meningococcal disease, either by culture or serology ( 13 group B, nine group $\mathrm{C}$, grouping unavailable in two); seven had pneumococcal disease; one had group B streptococcal meningitis. Blood cultures were positive in 10 cases, CSF in six, and both blood and CSF in five; a retrospective serological diagnosis of meningococcal disease was made in 11 cases.

(2) Set 2 consisted of 19 patients with clinically documented meningococcal disease (shock, petechial rash notified to the consultant for communicable disease control and close contacts given prophylaxis). CSF specimens were obtained from only two patients in this group. Blood cultures were received from 17 patients. Throat swabs were processed from nine patients and skin biopsies were cultured from an additional two patients. All cultures were negative, but many patients had received previous antibiotics.

(3) Set 3 contained 29 patients admitted with meningitis/meningism not considered to be bacterial in origin and one patient with possible bacterial meningitis. Specimens from two patients with a diagnosis of overwhelming postsplenectomy infection (OPSI), and three patients with lobar pneumonia were included. All microbiological investigations were negative.

(4) Set 4 (controls) contained 10 urines collected from healthy volunteers and antenatal patients and seven control sera from hospital inpatients with unrelated conditions. Specimens from six study patients who were ultimately diagnosed as having unrelated conditions (including group A streptococcal infections, drug overdose, viral infections, and inborn errors of metabolism) were included in this group.

In all, 128 specimens (52 urine, 60 serum, and $16 \mathrm{CSF}$ ) were collected from 109 individuals. Where the volume of urine was sufficient $(>8 \mathrm{ml})$, a $6 \mathrm{ml}$ aliquot was concentrated 15-fold in a Minicon B-15 concentrator (Amicon) and the neat and concentrated specimens were tested separately. This yielded an additional 32 specimens for testing.

\section{TEST PROCEDURES}

Specimens were prepared according to the manufacturer's instructions (Wellcogen kit; Murex Diagnostics). Samples were filtered before following the standard test card procedure. Specimens were assessed against the range of antibody coated test particles. For ultrasonic enhancement, reaction droplets consisting of $25 \mu \mathrm{l}$ of test or control sample and 25 $\mu 1$ of appropriate latex suspension were mixed on a solid non-adsorbing surface and drawn into a $2 \mathrm{~mm}$ internal diameter glass capillary tube using a syringe. The capillary tube was located on the axis of a PCA4 tubular lead zirconate titanate transducer driven at $4.5 \mathrm{MHz}$, such that the droplet was exposed to ultrasound along its entire length. Further details of the standing wave pattern around the transducer axis have been given elsewhere. ${ }^{6}$

Ultrasonic exposure time was one minute, after which the droplet was expelled from the capillary tube onto a test card, drawn into a slide of rectangular cross section, and examined for agglutination by microscopy.

\section{Results}

SET 1: CONFIRMED BACTERIAL MENINGITIS OR SEPSIS

In patients with meningococcal disease, the test card was positive in $15 / 35$ specimens (43\%), detecting only $13 / 24$ patients $(54 \%)$ compared to $18 / 24$ patients $(75 \%)$ following ultrasonic enhancement (table 2). CSF was the only specimen to approach diagnostic usefulness using the standard test card procedure. Following ultrasonic enhancement, the sensitivity of detection in the CSF rose markedly, as did detection in the serum. Neat urine showed no enhancement and yielded one false positive result in a patient with documented meningococcal group $\mathrm{B}$ disease who gave a positive result for streptococcal group B antigen on both test card and ultrasound enhancement. This was attributed to contamination of the urine specimen. All other antigen results were concordant with the meningococcal group (where known).

Seven patients had pneumococcal disease. The latex agglutination test performed poorly in this group and was negative by test card procedure in all cases; it detected only one case of meningitis after enhancement.

One patient had group B streptococcal meningitis. This patient's urine was negative using the test card procedure, but ultrasonic enhancement correctly identified the causative organism.

SET 2: CLINICAL MENINGOCOCCAL SEPSIS

Table 2 shows the comparative performance between the test card procedure and ultrasonic enhancement in these patients. The test card performed poorly and only three of 19 cases 
Table 2 Meningococcal disease: comparison of test card and ultrasonic enhancement

\begin{tabular}{|c|c|c|c|c|c|c|c|c|}
\hline \multirow[b]{2}{*}{ Positive agglutination specimens (patients) } & \multicolumn{4}{|c|}{ Test card } & \multicolumn{4}{|c|}{ Ultrasound } \\
\hline & $\begin{array}{l}\text { Urine } \\
\text { (neat) }\end{array}$ & $\begin{array}{l}\text { Urine } \\
\text { (conc) }\end{array}$ & Serum & $C S F$ & $\begin{array}{l}\text { Urine } \\
\text { (neat) }\end{array}$ & $\begin{array}{l}\text { Urine } \\
\text { (conc) }\end{array}$ & Serum & $C S F$ \\
\hline \multicolumn{9}{|l|}{ Confirmed meningococcal, $\mathrm{n}=35(24)$} \\
\hline Group B & $1 / 5$ & $1 / 4$ & $1 / 4$ & $3 / 7$ & $1 / 5$ & $1 / 4$ & $2 / 4$ & $6 / 7$ \\
\hline Group C & $2 / 4$ & NA & $3 / 6$ & $2 / 2$ & $2 / 4$ & NA & $5 / 6$ & $2 / 2$ \\
\hline Not grouped & NA & NA & $0 / 1$ & $2 / 2$ & NA & NA & $0 / 1$ & $2 / 2$ \\
\hline Subtotal & $3 / 9$ & $1 / 4$ & $4 / 11$ & $7 / 11$ & $3 / 9$ & $1 / 4$ & $7 / 11$ & $10 / 11$ \\
\hline Total specimens (patients) & \multicolumn{4}{|c|}{$15 / 35(13 / 24)$} & \multicolumn{4}{|c|}{$21 / 35(18 / 24)$} \\
\hline Clinically documented meningococcal disease $n=32(19)$ & $1 / 13$ & $1 / 8$ & $2 / 9$ & $0 / 2$ & $4 / 13$ & $6 / 8$ & $7 / 9$ & $0 / 2$ \\
\hline Total specimens (patients) & \multicolumn{4}{|c|}{$4 / 32(3 / 19)$} & \multicolumn{4}{|c|}{$17 / 32(14 / 19)$} \\
\hline Total specimens & \multirow{2}{*}{\multicolumn{4}{|c|}{$\begin{array}{l}19 / 67(29 \%) \\
16 / 43(37 \%)\end{array}$}} & \multicolumn{4}{|c|}{$38 / 67(57 \%)$} \\
\hline Total patients & & & & & \multicolumn{4}{|c|}{$32 / 43(74 \%)$} \\
\hline
\end{tabular}

Conc $=$ concentrated; $\mathrm{NA}=$ not available

$(16 \%)$ gave positive results for meningococcal antigenaemia, all reacting with ACY. After ultrasonic enhancement, this rose to 14 cases (74\%; eight ACY, six BK1). CSF specimens were negative in the two cases where they were available. Concentrated urine specimens and serum showed the best enhancement.

\section{SET 3: POSSIBLE MENINGITIS AND RELATED}

CONDITIONS

All specimens from patients with a diagnosis of possible meningitis were negative, apart from two cases which gave ambivalent results after ultrasonic enhancement, both with group B antibody coated particles. Both equivocal results occurred on the same day and these specimens were negative on retesting one week later. Of the two patients with OPSI, one gave a positive result for pneumococcal disease and the other for meningococcal B disease. All specimens from the three patients with lobar pneumonia were negative.

SET 4: CONTROLS

All specimens were negative by both test card and ultrasound.

\section{Discussion}

In summary, diagnosis of the 43 patients with meningococcal disease based on antigen detection rose from $37 \%$ of cases using the standard test card to $74 \%$ after ultrasonic enhancement. Sensitivity approached that reported for PCR techniques at a fraction of the cost and time involved for the latter. Enhancement was maximal in the patients from set 2 with clinically diagnosed but culture negative meningococcal disease, most of whom had received antibiotics before specimens were taken. Diagnosis increased from $16 \%$ to $74 \%$ of cases in this group where the clinical impact of rapid diagnosis is likely to be maximal.

Some enhancement was also seen in the few cases of bacterial meningitis due to other causes (S pneumoniae and group B streptococci) but the numbers of specimens are too small for any firm conclusions to be drawn. The results for $S$ pneumoniae were disappointing. Urine specimens were consistently negative, even in cases of documented septicaemia. Suspected cases presenting with lobar pneumonia were similarly negative. The results provide no support for the diagnostic usefulness of antigen detection in this condition or the recommendations of the
British Thoracic Society that urine should be examined for antigen to confirm pneumococcal pneumonia. ${ }^{9}$ It is possible that the large complex polysaccharide antigens are broken down before excretion in the urine. This and the time course of antigenuria following meningitis, septicaemia, or pneumonia needs further investigation. Extreme care should be taken to avoid contamination of urine specimens and false positive results. ${ }^{10}$

At the present time, commercial antigen detection kits contribute little to the diagnosis of meningococcal disease. Ultrasonic enhancement is likely to be particularly useful in confirming clinical meningococcal sepsis in patients who have received previous antibiotics. It can give rapid preliminary information on the group involved, which may be important in outbreak management. It can be used to determine the need for continued intravenous antibiotics after 48 hours in a well child with a rash and negative cultures.

This preliminary study on 168 specimens from 109 individuals suggests that ultrasonic enhancement is a useful tool. Further studies are being undertaken to establish the clinical impact in management of patients with meningococcal disease.

This work was supported by the Meningitis Research Foundation.

1 Jorden JZ, Slack MPE. Haemophilus influenzae: then and now. Eur F Clin Microbiol Infect Dis 1995;14:935-48.

2 Fallon RJ, Madeley CR, Mayon-White RT, Urquhart AM, Welsby PD, and The Research Committee for the BSSI. Bacterial meningitis: causes for concern. F Infect 1994;30: 89-94.

3 Bhisikul DM, Hogan AE, Tanz RR. The role of bacterial antigen tests in the diagnosis of bacterial meningitis. Pediatr Emerg Care 1994;10:67-71.

4 Newcombe J, Cartwright K, Palmer WH, et al. PCR of peripheral blood for the diagnosis of meningococcal disease. $\mathcal{F}$ Clin Microbiol 1996;34:1637-40.

5 Perkins MD, Mirrett S, Reller LB. Rapid bacterial antigen detection is not clinically useful. $\mathcal{F}$ Clin Microbiol 1995;33: 1486-91.

6 Grundy MA, Moore K, Coakley WT. Increased sensitivity of diagnostic latex agglutination tests in an ultrasonic standing wave field. F Immunol Methods 1994;176:169-77. Grundy MA, Barnes RA, Coakley WT. Highly sensitive detection of fungal antigens by ultrasound-enhanced latex agglutination. F Med Vet Mycol 1995;33:201-3.

8 Coakley WT, Jenkins P, Barnes RA. Ultrasound-increased sensitivity of coated particle agglutination detection of meningitis antigens in buffer and body fluids. F Immunol Methods 1997;205:191-200.

9 Pneumonia Subcommittee of the British Thoracic Society. Guidelines for the management of community-acquired pneumonia in adults admitted to hospital. $\mathrm{Br} \mathcal{F}$ Hosp Med 1993;49:346-50

10 Boyer D, Gordon RC, Baker T. Lack of clinical usefulness of a positive latex agglutination test for Neisseria meningitidis/Escherichia coli antigens in the urine. Pediatr Infect Dis F 1993;12:779-80. 